\title{
SGLT2 promotes cardiac fibrosis following myocardial infarction and is regulated by miR-141
}

\author{
GANG LI, CONGCHUN ZHAO and SHANHUA FANG
}

\begin{abstract}
Department of Geriatrics, The Second Affiliated Hospital of Wannan Medical College, Wuhu, Anhui 241000, P.R. China
\end{abstract}
Received February 21, 2020; Accepted October 2, 2020

DOI: $10.3892 /$ etm.2021.10147

\begin{abstract}
Cardiac fibrosis is a primary event during myocardial infarction (MI) progression, which impairs cardiac function. The present study aimed to investigate the effect of SGLT2 on cardiac fibrosis following MI. To validate the role of SGLT2 in the regulation of cardiac fibrosis in vivo, an MI rat model was established. Echocardiography was performed to determine cardiac function at 4 weeks post-MI. MI model rats were transfected with short hairpin RNA (sh)-SGLT2 or sh-negative control lentiviruses to investigate the effect of SGLT2 on rat heart function post-MI. Subsequently, the effects of SGLT2 on the cardiac fibrosis of infarcted hearts were assessed by performing Masson's trichrome staining. To further clarify the effect of SGLT2 on cardiac fibroblast proliferation, TGF $\beta$ was used to stimulate primary cardiac fibroblasts in vitro. The results demonstrated that SGLT2 served a key role in cardiac fibrosis. SGLT2 expression levels in infarct tissues were significantly increased at week 1 post-MI compared with the sham group. Compared with the control group, SGLT2 knockdown attenuated cardiac fibrosis by inhibiting the expression of collagen I and collagen III in cardiac fibroblasts in vitro and in vivo. Furthermore, the results indicated that SGLT2 expression was modulated by miR-141 in cardiac fibroblasts. In summary, the present study indicated that upregulated SGLT2 expression in cardiac fibrosis following MI was regulated by miR-141 and SGLT2 that knockdown reduced cardiac fibrosis and improved cardiac function after MI.
\end{abstract}

\section{Introduction}

Globally, myocardial infarction (MI) is a leading cause of morbidity and mortality, and causes progressive deterioration that ultimately results in heart failure (1). Cardiac fibrosis is a primary event in MI progression, and is characterized by

Correspondence to: Dr Gang Li, Department of Geriatrics, The Second Affiliated Hospital of Wannan Medical College, 10 Kangfu Road, Wuhu, Anhui 241000, P.R. China

E-mail: ligangmedical@sina.com

Key words: myocardial infarction, cardiac fibrosis, sodium-glucose linked transporter 1, microRNA-141 the transformation of fibroblasts into myofibroblasts and the production of excessive extracellular matrix proteins, including collagen I and III, within the myocardium (2). During the chronic stages of MI, abnormal cardiac fibrosis inevitably causes the excessive production of extracellular matrix proteins and decline in cardiac function $(2,3)$. Therefore, novel strategies are required to inhibit cardiac fibrosis to improve heart function in patients with MI.

Sodium-glucose linked transporter (SGLT) 1 and SGLT2 are primary SGLTs that contribute to the reabsorption of kidney-filtered glucose $(4,5)$. A previous study demonstrated that SGLT1 was involved in cardioprotection against ischemia-reperfusion injury (6). By contrast, SGLT2 inhibitors have been reported to decrease blood glucose independently and reduce the risk of severe heart failure (7). Furtado et al (8) demonstrated that dapagliflozin, an SGLT2 inhibitor, markedly reduced the risk of both major adverse cardiovascular events and cardiovascular death/hospitalization for heart failure in patients with type 2 diabetes mellitus and previous MI. Moreover, to the best of our knowledge, dapagliflozin is the only SGLT-2 inhibitor that reduces cardiac necrosis and the worsening of heart failure $(8,9)$. Empagliflozin, an SGLT-2 inhibitor, has been studied in a clinical trial and the results demonstrated reduced cardiovascular mortality of patients with type 2 diabetes mellitus (10). Furthermore, SGLT2 inhibition with empagliflozin effectively improved cardiac diastolic function in a female rodent model of diabetes (11). Ye et al (12) demonstrated that the inhibition of SGLT-2 reduced NLR family pyrin domain containing 3 (Nlrp3)/apoptosis-associated speck-like protein (ASC) inflammasome activation and attenuated the development of diabetic cardiomyopathy in mice. Additionally, dapagliflozin, a selective SGLT2 inhibitor, served a protective role in cardiac fibrosis in infarcted rat hearts (11). The aforementioned previous studies demonstrated that SGLT2 served a potential role in the pathogenesis of heart disease. However, the biological function of SGLT2 in cardiac fibrosis is not completely understood.

MicroRNAs (miRNAs/miRs) have been reported to be involved in the regulation of cardiac fibrosis. miR-21, miR-34, miR-199b and miR-208 have been identified to contribute to myocardial fibrosis and are upregulated in MI (13). By contrast, miR-1, miR-29, miR-133a and miR-214 are antifibrotic miRNAs (13). However, the mechanism underlying miRNA-mediated regulation of cardiac fibrosis in MI is not completely understood. 
The present study aimed to investigate the role of SGLT2 in cardiac fibrosis following MI. Moreover, whether upregulated SGLT2 levels in cardiac fibrosis following MI are regulated in a miRNA dependent manner was also investigated.

\section{Materials and methods}

Animals, MI model and assessment of heart function. A total of 65 , six to eight-week male Sprague-Dawley rats (weight, 200-300 g) were purchased from the Academy of Military Medical Sciences. The rats were kept in a temperature-controlled room, with a humidity of $40-70 \%$, in a $12 \mathrm{~h}$ light-dark cycle with free access to standard chow and tap water. All animals were reared in a specific pathogen-free environment at a comfortable temperature and humidity. All experimental procedures were approved by the Animal Ethics Committee of the Second Affiliated Hospital of Wannan Medical College, Wuhu, China (approval no. DWL-1804-007).

MI was modeled in rats via the permanent ligation of the left anterior descending branch of the coronary artery with prolene sutures, as previously described (14). Briefly, rats were anesthetized intraperitoneally with $40 \mathrm{mg} / \mathrm{kg}$ sodium pentobarbital. The thoracic cavities were opened and the left anterior descending (LAD) coronary arteries were permanently ligated with a 7-0 polypropylene suture. In the sham operation group, animals underwent the same procedure, except the LAD was left untied. Following euthanasia by anesthetic overload with intraperitoneal $90 \mathrm{mg} / \mathrm{kg}$ ketamine and $10 \mathrm{mg} / \mathrm{kg}$ xylazine, the infarct zones and far zones of the hearts were quickly excised for the detection of RNA, protein and fibrosis levels. The SGLT2 and miR-141 expression was measured at infarct zones at 1,2, 3 and 4 weeks post-MI. To evaluate the effect of SGLT2 on MI in vivo, lentiviruses containing sh-SGLT2 or sh-NC were obtained from Shanghai GenePharma Co., Ltd. Animals were divided into the following four groups: i) sham $(\mathrm{n}=5)$; ii) MI ( $=5)$; iii) MI + short hairpin RNA (sh)-negative control $(\mathrm{NC}, \mathrm{n}=5)$; and iv) $\mathrm{MI}+\mathrm{sh}-\mathrm{SGLT} 2(\mathrm{n}=5)$. Following LAD ligation, $10^{8}$ PFU of sh-SGLT2, sh-NC or PBS (100 $\left.\mu \mathrm{l}\right)$ was intramyocardially injected into the corresponding groups.

Echocardiography was performed to determine cardiac function at 4 weeks post-MI using a Vevo 2100 system (VisualSonics, Inc.) with an $80 \mathrm{MHz}$ probe. Left ventricular parameters were recorded from two-dimensional images using the M-mode interrogation in the short-axis view.

Masson trichrome staining. At 4 weeks post-MI, heart sections from MI model rats were excised from an area perpendicular to the axis of the LAD coronary arteries. Briefly, the tissues were fixed in $4 \%$ of paraformaldehyde for $24 \mathrm{~h}$ at room temperature, embedded in paraffin and $5 \mu \mathrm{m}$ sections were taken. Sections were then stained using a Masson's Trichrome stain kit (cat. no. 1004850001; Sigma-Aldrich; Merck KGaA,) according to the manufacturer's protocol. All of the images (magnification, x12.5) were captured on a confocal microscope (Nikon Corporation). Average ratios of the fibrotic areas to the entire left ventricular cross-sectional area were analyzed using ImageJ 1.48u software (National Institutes of Health).

Primary cardiac fibroblasts isolation and culture. Cardiac fibroblasts were isolated as previously described (15). Briefly, primary cardiac fibroblasts were isolated from 1-3 day-old female Sprague-Dawley rats $(n=3)$. The female neonatal rats used in this experiment were bred by ourselves, which were co-housed with the maternal rat since birth. Their parents housing conditions are aforementioned. Cardiac fibroblasts were cultured in DMEM (Gibco; Thermo Fisher Scientific, Inc.) containing 10\% FBS (Gibco; Thermo Fisher Scientific, Inc.), $100 \mathrm{U} / \mathrm{ml}$ penicillin and $100 \mu \mathrm{g} / \mathrm{ml}$ streptomycin (Sigma-Aldrich, Merck $\mathrm{KGaA}$ ) at $37^{\circ} \mathrm{C}$ with $5 \% \mathrm{CO}_{2}$. Myocardial fibrosis phenotypes were induced using $20 \mathrm{ng} / \mathrm{ml}$ recombinant TGF- $\beta$ (PeproTech, Inc.) at $37^{\circ} \mathrm{C}$ for $72 \mathrm{~h}$, as previously described (16).

Cell infection and transfection. Lentiviruses containing sh-SGLT2 or sh-NC were obtained from Shanghai GenePharma Co., Ltd. For lentiviral infection, primary cardiac fibroblasts were incubated with sh-SGLT2 or sh-NC at an optimal multiplicity of infection of 15 at $37^{\circ} \mathrm{C}$ for $12 \mathrm{~h}$. Subsequently, the medium was removed and cells were incubated in complete medium (DMEM containing 10\% FBS, $100 \mathrm{U} / \mathrm{ml}$ penicillin and $100 \mu \mathrm{g} / \mathrm{ml}$ streptomycin) at $37^{\circ} \mathrm{C}$ for $72 \mathrm{~h}$. Infection efficiency was assessed via reverse transcription-quantitative PCR and western blotting. miR-141 mimics (miR-141 mimics: 5'-UAACACUGUCUGGUAAAGAUGG-3') and scrambled non-coding RNAs (miR-141 NC: 5'-ACGUGACACGUUCGG AGAATT-3') were purchased from Ambion (Thermo Fisher Scientific, Inc.). pcDNA3.1 vectors containing full-length SGLT2 cDNA sequences (pcDNA3.1-SGLT2) were purchased from Shanghai GenePharma Co., Ltd. Primary cardiac fibroblasts $\left(3 \times 10^{5}\right)$ were transfected with 20 pmol miR-141 mimics, 20 pmol miR-141 NC, $1 \mu \mathrm{g}$ pcDNA3.1-SGLT2 or $1 \mu \mathrm{g}$ empty control vector using Lipofectamine ${ }^{\circledR} 2000$ (Invitrogen; Thermo Fisher Scientific, Inc.) in serum-free medium. Control experiments were performed with mock-transfected cells using the same procedure. After $8 \mathrm{~h}$ of transfection at $37^{\circ} \mathrm{C}$, all of the transfected and mock-transfected primary cardiac fibroblasts were transferred to DMEM with $10 \%$ FBS for an additional $24 \mathrm{~h}$ and incubated at $37^{\circ} \mathrm{C}$. Following this incubation, the subsequent experiments were conducted.

Reverse transcription-quantitative PCR (RT-qPCR). Total RNA was extracted from cardiac tissues and primary cardiac fibroblasts using TRIzol ${ }^{\circledR}$ reagent (Invitrogen; Thermo Fisher Scientific, Inc.). Total RNA was reverse transcribed into cDNA using a PrimeScript RT kit (Takara Bio, Inc.). The temperature protocol used for RT was $37^{\circ} \mathrm{C}$ for $15 \mathrm{~min}$ and $85^{\circ} \mathrm{C}$ for $15 \mathrm{sec}$. The samples were then kept at $4^{\circ} \mathrm{C}$ for immediate use or $-20^{\circ} \mathrm{C}$ for long term storage. Subsequently, qPCR was carried out using the Power SYBR GREEN PCR master mix (Takara Bio, Inc.) with ABI7300 detector (Applied Biosystems; Thermo Fisher Scientific, Inc.). The reaction parameters of standard procedure for two-step amplification were as follows: $95^{\circ} \mathrm{C}$ for $10 \mathrm{~min}$, and 40 cycles at $95^{\circ} \mathrm{C}$ for $15 \mathrm{sec}$ and $60^{\circ} \mathrm{C}$ for $30 \mathrm{sec}$. The following primer sequences were used: SGLT2 forward, 5'-GCAGAAGGTCCTGATTGATA-3' and reverse, 5'-GCG ATGACAGAAGCGTAAA-3'; collagen I forward, 5'-CGAGTA TGGAAGCGAAGGT-3' and reverse, 5'-CCACAAGCGTGC TGTAGGT-3'; collagen III forward, 5'-CCACCCTGAACT CAAGAGC-3' and reverse, 5'-TGAACTGAAAGCCACCAT T-3'; and $\beta$-actin forward, 5'-GTAAAGACCTCTATGCCA 


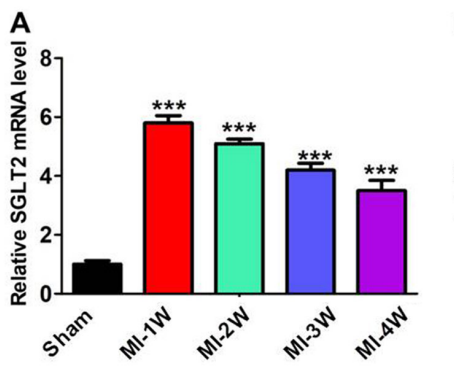

B
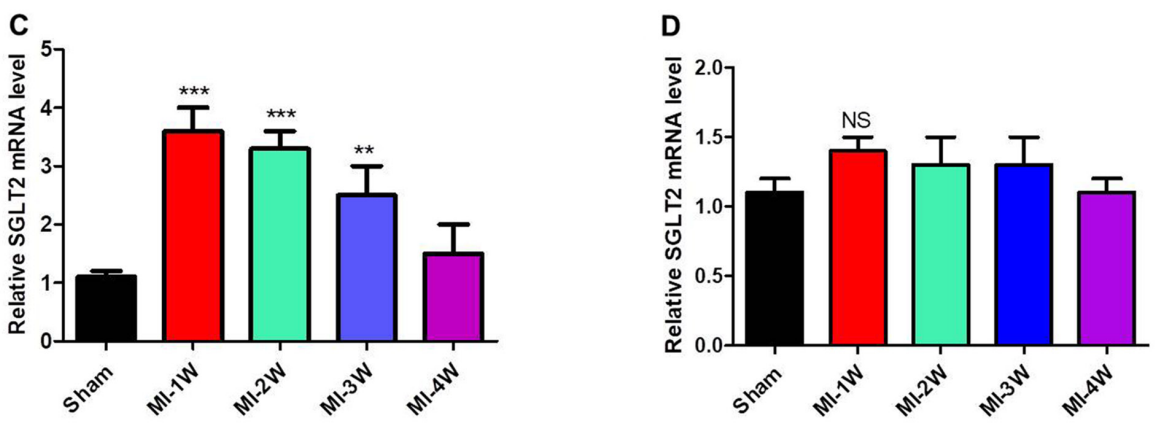

Figure 1. SGLT2 expression was analyzed in the infarcted myocardial tissues of MI model rats. SGLT2 (A) mRNA and (B) protein expression levels in infarcted areas of MI model rats at indicated times ( $n=5)$. SGLT2 mRNA expression levels in the (C) border and (D) far zones $(n=5) .{ }^{* *} P<0.01,{ }^{* * * *} P<0.001$ vs. sham. SGLT2, sodium-glucose linked transporter 2; MI, myocardial infarction; W, week; NS, not significant.

ACA-3' and reverse, 5'-GGACTCATCGTACTCCTGCT-3'; U6 forward, 5'-CTCGCTTCGGCAGCACA-3'; and reverse, 5'-AACGCTTCACGAATTTGCGT-3'. Primers were provided by Ambion (Thermo Fisher Scientific, Inc.). miR-141 levels were normalized to U6. mRNA and miRNA expression levels were quantified using the $2^{-\Delta \Delta \mathrm{Cq}}$ method (17) and normalized to the internal reference genes $\beta$-actin and U6, respectively.

Bioinformatics. miRNA binding sites were predicted using TargetScan (v7.0; targetscan.org) (18-20).

Western blotting. Total protein was extracted from primary cardiac fibroblasts and cardiac tissues using Laemmli sample buffer (Bio-Rad Laboratories, Inc.). Protein concentrations were determined using a BCA protein assay kit (Thermo Fisher Scientific, Inc.). Proteins $(30 \mu \mathrm{g})$ were separated using a $10 \%$ SDS-PAGE gel and transferred to PVDF membranes (Roche Diagnostics), which were blocked with $5 \%$ non-fat milk at $25^{\circ} \mathrm{C}$ for $1 \mathrm{~h}$. Subsequently, the membranes were incubated overnight at $4^{\circ} \mathrm{C}$ with the following primary antibodies: Anti-SGLT2 (cat. no. ab37296; 1:1,000, Abcam), anti-collagen I (cat. no. ab34710; 1:1,000, Abcam), anti-collagen III (cat. no. ab7778; 1:1,000, Abcam) and anti- $\beta$-actin (cat. no. ab8227; 1:1,000, Abcam). The secondary antibody (HRP-conjugated; cat. no. ab7090; Abcam) was diluted to a ratio of 1:5,000 and incubated for $1 \mathrm{~h}$ at $25^{\circ} \mathrm{C}$. Protein bands were visualized using enhanced chemiluminescence (Thermo Fisher Scientific, Inc.). $\beta$-actin was used as the loading control.

Cell viability assay. An MTT assay was performed to assess cell viability, as previously described (21). Briefly, primary cardiac fibroblasts $\left(5 \times 10^{3}\right)$ were seeded into 96 -well plates and treated with designated treatments. After $48 \mathrm{~h}, 15 \mu \mathrm{l}$ MTT solution (Sigma-Aldrich; Merck KGaA) was added to each well and incubated at $37^{\circ} \mathrm{C}$ for $4 \mathrm{~h}$. Subsequently, $200 \mu \mathrm{l}$
DMSO was added into each well to dissolve the formazan. Absorbance was measured at a wavelength of $490 \mathrm{~nm}$ using a microplate reader (Tecan Austria $\mathrm{GmbH}$ ).

Luciferase reporter assay.SGLT23'-untranslated region(UTR) sequences were inserted into pmiR-RB-REPORT vectors (Guangzhou Ribobio Co., Ltd.). SGLT2 3'-UTR-mutants were generated in which 6 complementary binding site nucleotides were altered. 293T cells ( $4 \times 10^{5}$ cells) were co-transfected with SGLT23'-UTR-wild-type (1 $\mu \mathrm{g})$ or SGLT2 3'-UTR-mutant $(1 \mu \mathrm{g})$ and miR-141 mimics (40 pmol) or miR-141 NC (40 pmol) using Lipofectamine ${ }^{\circledR} 2000$ (Invitrogen; Thermo Fisher Scientific, Inc.). After $48 \mathrm{~h}$, transfected cells were collected and luciferase activity was detected using a Luciferase Reporter kit (Promega Corporation). Firefly luciferase activity was normalized to Renilla luciferase activity.

Statistical analysis. Statistical analysis was carried out using GraphPad Prism software (version 5.01; GraphPad Software, Inc.). Data are presented as the mean $\pm \mathrm{SD}$. All experiments were performed at least in triplicate. Comparisons among groups were analyzed using a one-way ANOVA and Tukey's post hoc tests with GraphPad Prism software version 7.0 (GraphPad Software, Inc.). Comparisons between two groups were analyzed using an unpaired Student's t-test. $P<0.05$ was considered to indicate a statistically significant difference.

\section{Results}

SGLT2 expression is increased in the infarct myocardium post-MI in rats. To investigate the role of SGLT2 in the regulation of cardiac fibrosis in vivo, MI rat models were established. mRNA and protein expression levels of SGLT2 in cardiac tissues at infarct zones at 1, 2, 3 and 4 weeks post-MI were analyzed via RT-qPCR and western blotting (Fig. 1A and B). 

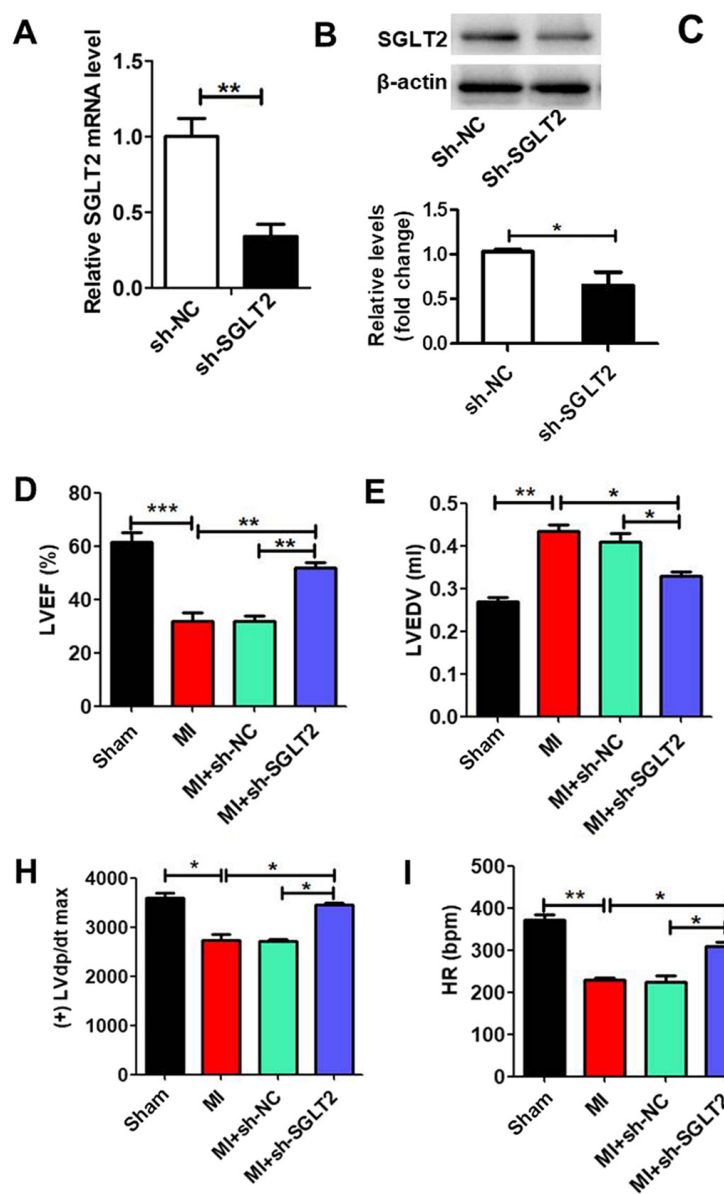
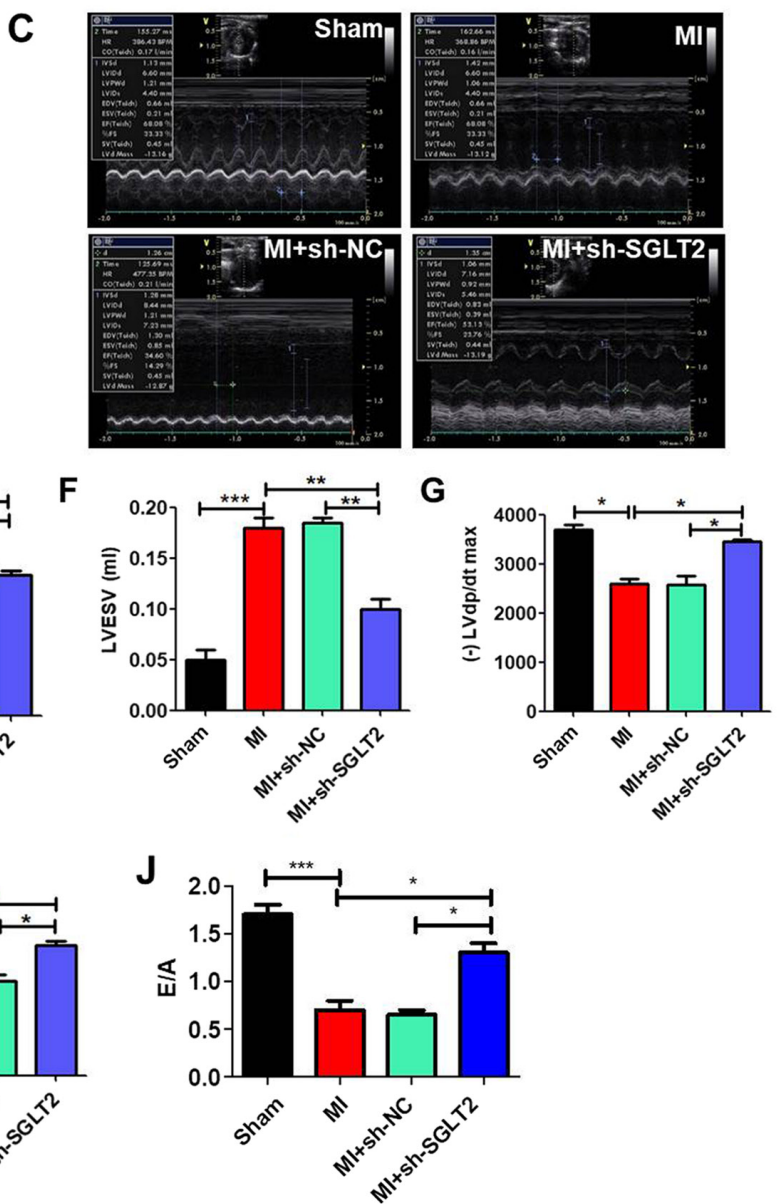

Figure 2. SGLT2 knockdown improves rat heart function following MI in vivo. SGLT2 (A) mRNA and (B) protein expression levels in infarcted areas treated with sh-SGLT2 or sh-NC (n=5). (C) Echocardiography results of rats in the different groups $(n=5)$. At 4 weeks post-MI, ventricular parameters were measured and analyzed by echocardiography, including (D) LVEF, (E) LVEDV, (F) LVESV, (G) (-) LVdp/dtmax, (H) (+) LVdp/dtmax, (I) HR and (J) E/A ratio (n=5). ${ }^{*} \mathrm{P}<0.05,{ }^{* *} \mathrm{P}<0.01,{ }^{* * * *} \mathrm{P}<0.001$. SGLT2, sodium-glucose linked transporter 2; MI, myocardial infarction; sh, short hairpin RNA; NC, negative control; LVEF, left ventricular ejection fractions; LVEDV, left ventricular end diastolic volume; LVESV, left ventricular end systolic volume; (-)/(+) LVdp/dtmax, the maximum left ventricular change in pressure/time; HR, heart rate; E/A ratio, ratio of the peak early transmitral flow velocity to peak late transmitral flow velocity.

A

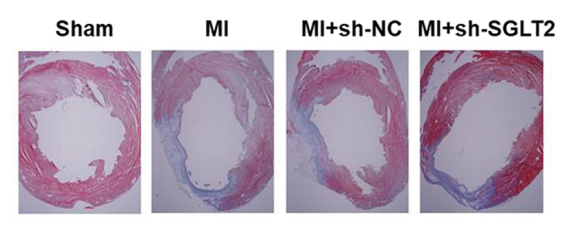

B

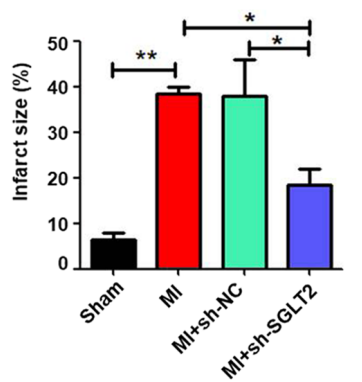

D
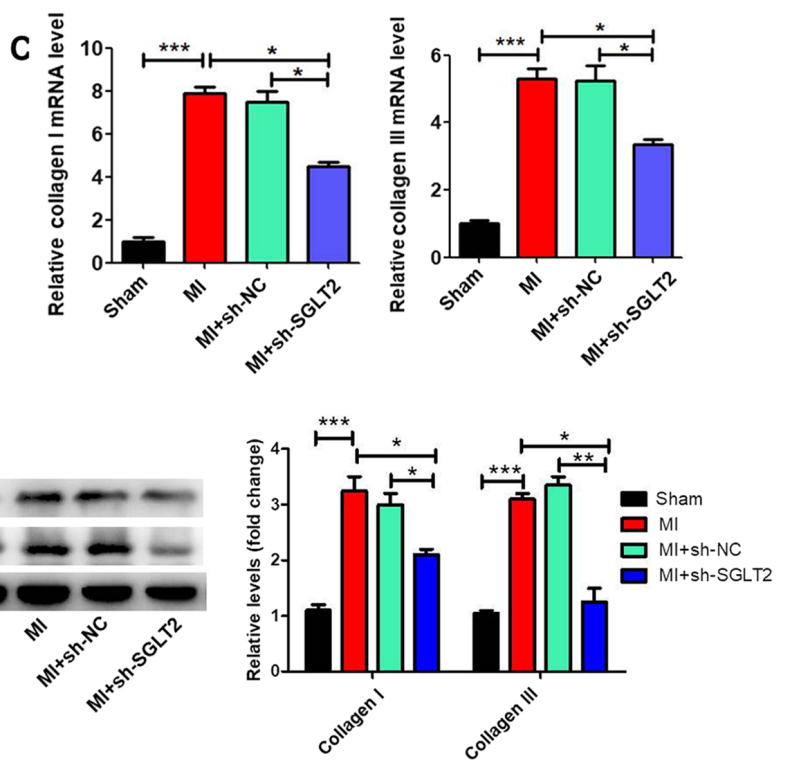

Figure 3. SGLT2 knockdown inhibits cardiac fibrosis following MI in vivo. (A) Representative photomicrographs of Masson trichrome staining of hearts of MI model rats treated with PBS, sh-NC or sh-SGLT2 ( $\mathrm{n}=5$, original magnification $\mathrm{x} 12.5$ ). (B) Fibrotic area was determined as the ratio of the average circumference of fibrotic scars (blue)/average inner circumference of the left ventricle ( $\mathrm{n}=5$ ). Collagen I and collagen III (C) mRNA and (D) protein expression levels in cardiac tissues $(\mathrm{n}=5) .{ }^{*} \mathrm{P}<0.05,{ }^{* * *} \mathrm{P}<0.01,{ }^{* * * *} \mathrm{P}<0.001$. SGLT2, sodium-glucose linked transporter 2; MI, myocardial infarction; sh, short hairpin RNA; NC, negative control. 

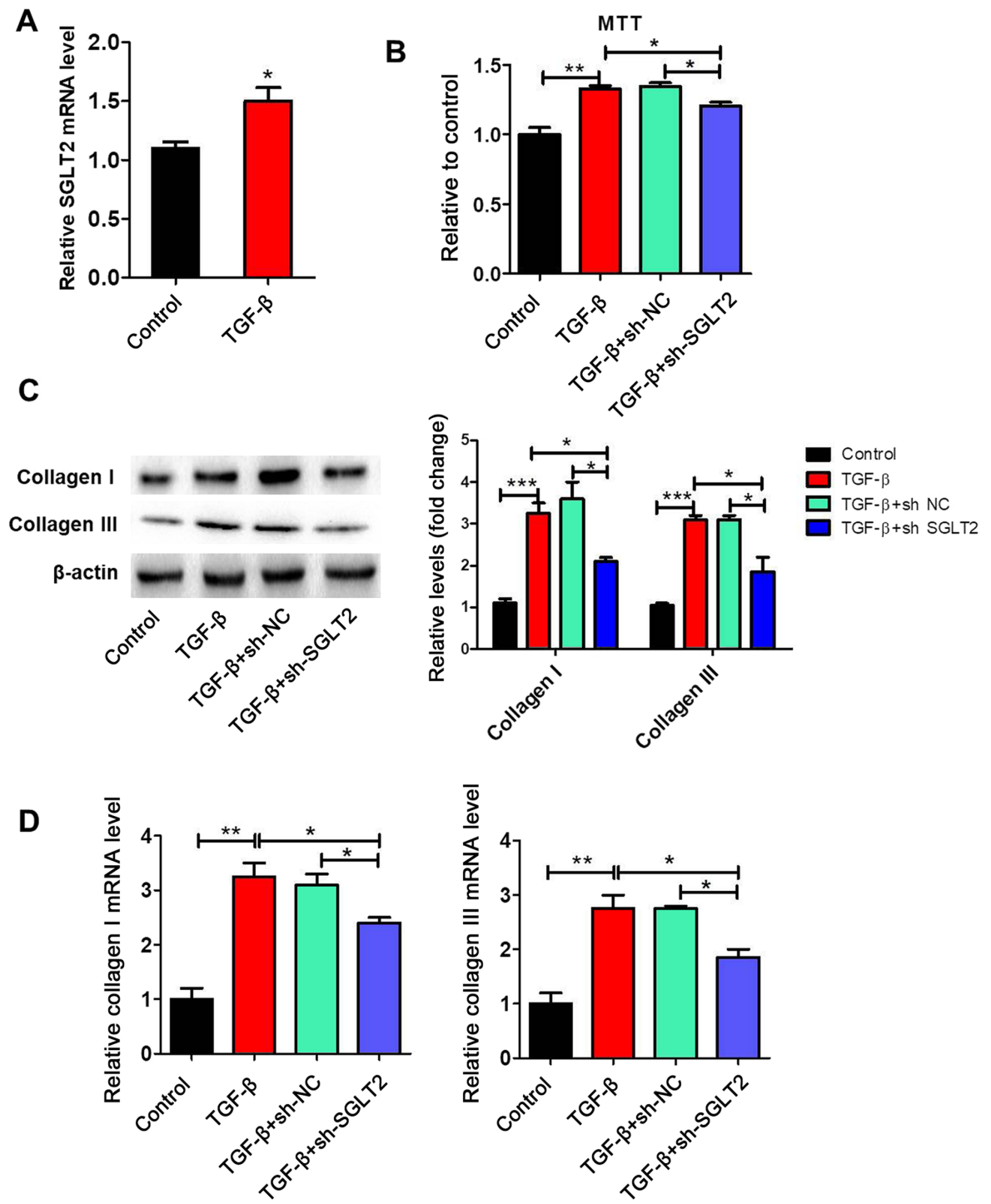

Figure 4. SGLT2 knockdown inhibits TGF $\beta$-induced proliferation and collagen synthesis in cardiac fibroblasts. Primary cardiac fibroblasts were transfected with sh-NC or sh-SGLT2 and then treated with $20 \mathrm{ng} / \mathrm{ml}$ recombinant TGF $\beta$ for $72 \mathrm{~h}$. (A) SGLT2 expression levels. (B) The MTT assay was performed to examine cell proliferation. (C) Protein and (D) mRNA expression levels and of collagen I and collagen III in primary cardiac fibroblasts. ${ }^{*} \mathrm{P}<0.05$, ${ }^{* *} \mathrm{P}<0.01$, ${ }^{* * *} \mathrm{P}<0.001$. SGLT2, sodium-glucose linked transporter 2; TGF $\beta$, transforming growth factor $\beta$; sh, short hairpin RNA; NC, negative control.

SGLT2 expression levels in MI model rats were significantly increased at week 1 post-MI compared with sham rats, with levels gradually decreasing with time. Furthermore, SGLT2 mRNA expression levels in the border and far zones, the zone of heart ventricular near the atril area, were analyzed. SGLT2 expression levels in the border zone (Fig. 1C) were similar to the expression levels in the infarct zone. In contrast, SGLT2 expression levels were not changed in the far zones of infarcts at 1,2, 3 and 4 weeks post-MI (Fig. 1D). The results indicated that SGLT2 served an important role in MI.

SGLT2 knockdown improves cardiac function following MI. MI model rats were treated with sh-SGLT2 or sh-NC lentiviruses to demonstrate the effect of SGLT2 on rat cardiac function following MI. sh-SGLT2 significantly reduced SGLT2 expression levels in MI tissues compared with the sh-NC group (Fig. 2A and B). Echocardiography was performed to evaluate left ventricular function in the different groups (Fig. 2C). Left ventricular mass indices, including left ventricular ejection fractions (Fig. 2D), left ventricular end diastolic volume (Fig. 2E), left ventricular end systolic volume (Fig. 2F), maximum left ventricular change in pressure over time $[(-) /(+)$ left ventricular diastolic pressure/dt maximum; Fig. 2G and H], heart rate (Fig. 2I) and E/A (ratio of the peak early transmitral flow velocity to peak late transmitral flow velocity; Fig. 2J) were analyzed to determine cardiac function. The results indicated that sh-SGLT2 enhanced heart function following MI.

SGLT2 knockdown attenuates cardiac fibrosis following MI. Masson's trichrome staining was performed to assess the effects of SGLT2 on cardiac fibrosis of infarcted hearts. Treatment with sh-SGLT2 decreased the fibrotic region (the blue region) and significantly decreased the infarct size compared with the MI or sh-NC-treated control groups (Fig. 3A and B). Compared with the sham group, MI significantly increased the expression of collagen I and collagen III (Fig. 3C and D), which was reversed by sh-SGLT2. 

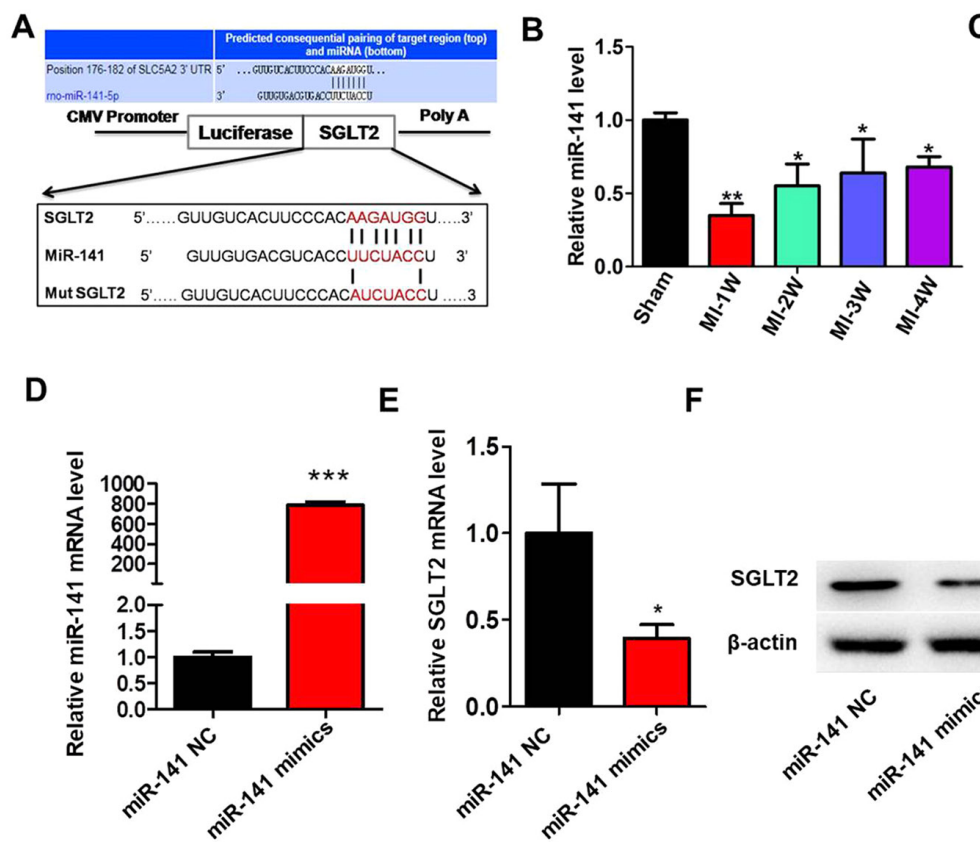

$\mathbf{F}$

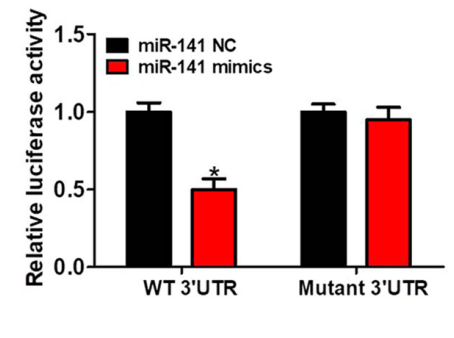

Figure 5. SGLT2 is targeted by miR-141. (A) The binding site between the 3'-UTR of SGLT2 mRNA and miR-141. SGLT2 3'-UTR-mutants were generated in which 6 complementary binding site nucleotides were altered. (B) miR-141 expression levels in the infarcted areas of MI model rats at indicated times. (C) Relative luciferase activities were determined by conducting a luciferase reporter assay. (D) Transfection efficiency of miR-141 mimics. SGLT2 (E) protein and (F) mRNA expression levels in primary cardiac fibroblasts transfected with miR-141 mimics. ${ }^{~} \mathrm{P}<0.05,{ }^{* *} \mathrm{P}<0.01,{ }^{* * *} \mathrm{P}<0.001$. SGLT2, sodium-glucose linked transporter 2; miR, microRNA; UTR, untranslated region; miR, microRNA; W, week; WT, wild-type; MUT, mutant; NC, negative control.

A

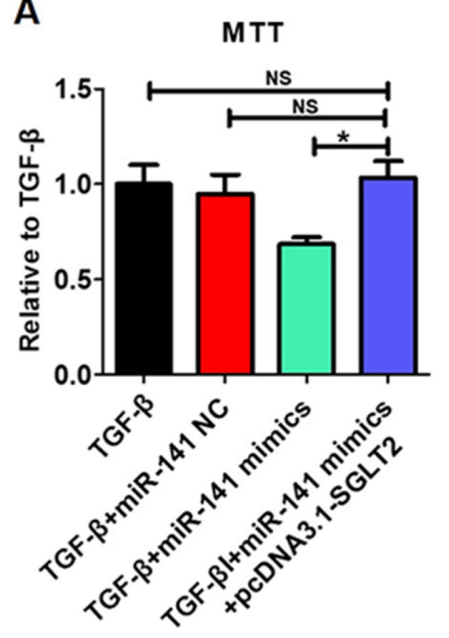

C

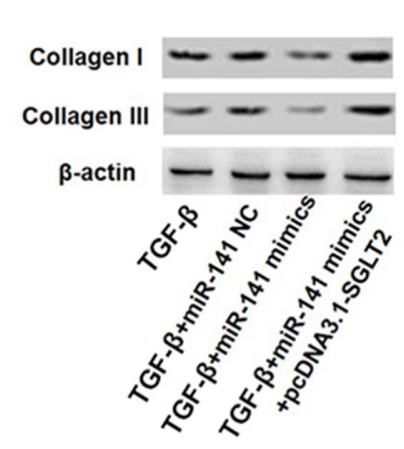

B
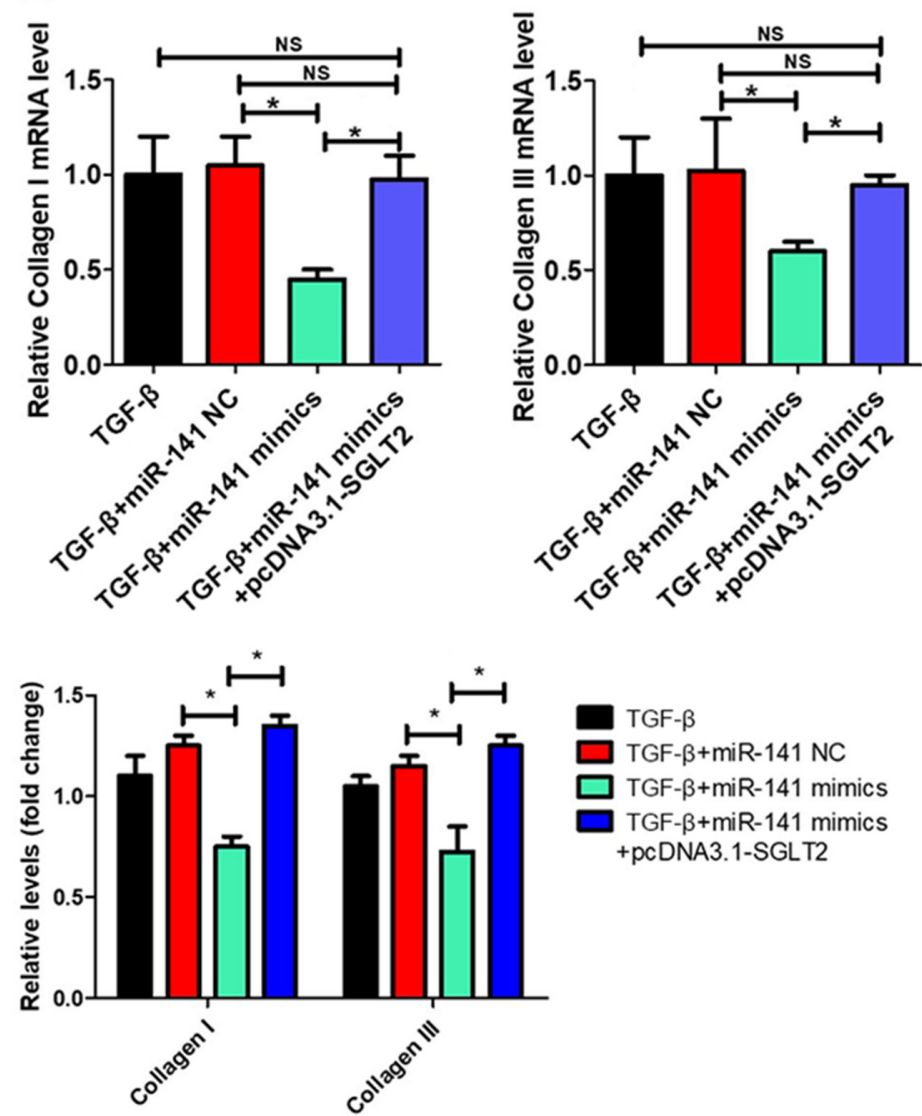

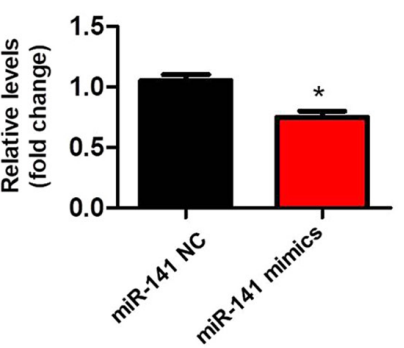


SGLT2 knockdown inhibits TGF $\beta$-induced proliferation and collagen synthesis in cardiac fibroblasts. SGLT2 expression levels were significantly increased by TGF $\beta$ treatment compared with the control group (Fig. 4A). To further clarify the effect of SGLT2 on cardiac fibroblasts proliferation, primary cardiac fibroblasts were stimulated with TGF $\beta$ in vitro. The MTT assay indicated that TGF $\beta$ significantly increased proliferation by $>1.3$ fold compared with the control group (Fig. 4B). TGF $\beta$-induced proliferation was significantly decreased by sh-SGLT2, but not by sh-NC. The protein and mRNA expression levels of collagen I and collagen III in cardiac fibroblasts were assessed via western blotting and RT-qPCR, respectively. The protein (Fig. 4C) and mRNA (Fig. 4D) expression levels of collagen I and collagen III were significantly upregulated in the TGF $\beta$ group compared with the control group. Furthermore, TGF $\beta$-induced collagen I and collagen III expression was significantly reversed by sh-SGLT2.

SGLT2 is targeted by miR-141. To identify why SGLT2 exhibited aberrant expression in the cardiac tissue infarct zones following MI, the present study hypothesized that dysregulated miRNAs may regulate SGLT2 expression during MI. A previous study reported that miR-141 was decreased in diabetic mice myocardium and cardiac fibroblasts treated with angiotensin II (22). TargetScan (v7.0; targetscan.org) was used to predict miR-141 binding sites in the 3'-UTR of SGLT2 (Fig. 5A). miR-141 expression levels in MI model rats were significantly decreased at 1 week post-MI compared with sham rats, but gradually increased with time (Fig. 5B). The results of the luciferase assay demonstrated that miR-141 overexpression significantly decreased the luciferase activity of the wild-type 3'-UTR compared with miR-141 NC. By contrast, miR-141 overexpression did not significantly alter the luciferase activity of mutant 3'UTR compared with miR-141 NC (Fig. 5C). Treatment with miR-141 mimics significantly increased miR-141 expression levels compared with miR-141 NC (Fig. 5D). Moreover, miR-141 overexpression significantly inhibited the mRNA (Fig. 5E) and protein (Fig. 5F) expression levels of SGLT2 compared with miR-141 NC.

TGF $\beta$-induced proliferation and collagen synthesis in cardiac fibroblasts is regulated by the miR-141/SGLT2 axis. The effects of the miR-141/SGLT2 axis on TGF $\beta$-induced proliferation and collagen synthesis in cardiac fibroblasts were examined. The transfection efficiency of pcDNA3.1-SGLT2 is presented in Fig. 6A. miR-141 overexpression significantly inhibited TGF $\beta$-induced cardiac fibroblast proliferation, which was reversed by SGLT2 overexpression (Fig. 6B). Additionally, miR-141 overexpression significantly decreased TGF $\beta$-induced collagen I and collagen III expression levels, whereas SGLT2 overexpression significantly reversed miR-141 overexpression-mediated effects (Fig. 6C).

\section{Discussion}

As a hallmark of MI, cardiac fibrosis is one of the most important factors that leads to heart failure $(23,24)$. Fibrosis is characterized by the accumulation of excess collagen, which causes cardiomyocyte dysfunction, abnormal differentiation of cardiac fibroblasts and interstitial fibrosis (25). Current antifibrotic drugs slow the progression of cardiac fibroblasts, but do not prevent or reverse progression (26). Therefore, it is crucial to identify specific targets for alternative therapeutic strategies.

SGLT2 inhibitors have been widely studied to improve cardiac diseases (10-12). Empagliflozin, an SGLT2 inhibitor, reduced cardiovascular mortality, as well as the occurrence of nonfatal MI and nonfatal strokes in patients with type 2 diabetes mellitus (10). Furthermore, empagliflozin or dapagliflozin, another SGLT-2 inhibitor, effectively improved cardiac diastolic function in a female rat model of diabetes (11). Additionally, SGLT-2 inhibition reduced the activation of the Nlrp3/ASC inflammasome and attenuated the development of diabetic cardiomyopathy in mice (12). The present study aimed to determine whether SGLT2 alleviated effects on the progression of cardiac fibrosis. The results demonstrated that SGLT2 levels in MI model rats were significantly increased at 1 week post-MI compared with sham rats, and continued to gradually decrease with time. Furthermore, SGLT2 knockdown improved cardiac function and attenuated cardiac fibrosis following $\mathrm{MI}$ in vivo compared with MI or sh-NC groups. SGLT2 knockdown also inhibited TGF $\beta$-induced proliferation and collagen synthesis in cardiac fibroblasts in vitro. Okada et al (27) demonstrated that dapagliflozin, an SGLT2 inhibitor, influenced interactions between SGLT2 and collagen I and IV, and established that discoidin domain receptor I served an important role in the adherence of collagen I and IV. Moreover, the expression levels of NLRP3, TNF $\alpha$ and caspase-1 were reduced in mice treated with dapagliflozin and the phosphorylated-adenosine monophosphate activated protein kinase (AMPK)/total-AMPK ratio was enhanced (12). The results of the aforementioned studies were similar to the results of the present study, which indicated that SGLT2 was associated with collagen deposition, collectively suggesting that SGLT2 served a key role in the pathogenesis of cardiac fibrosis.

miRNAs are 22-23 nucleotides noncoding RNA molecules that serve important roles in cardiovascular health and disease (28). For example, miR-98 downregulated TGF $\beta$ receptor 1 expression, ameliorated TGF- $\beta 1$-induced differentiation and inhibited collagen production of cardiac fibroblasts (29). Downregulation of miR-29 effectively increased the expression of collagens COL1A1, COL1A2 and COL3A1 in vitro and in vivo (30). Li et al (31) reported that miR-130a was increased in angiotensin II-infused mice, and promoted the expression of profibrotic genes and differentiation of myofibroblasts by inhibiting peroxisome proliferator activated receptor $\gamma$ expression (31). The present study demonstrated that miR-141 levels in MI model rats decreased at 1 week post-MI compared with sham rats, and gradually increased with time. It has been reported that miR-141 levels were decreased in diabetic mice myocardium and cardiac fibroblasts treated with angiotension II (22). The results of the present study indicated that miR-141 overexpression significantly inhibited TGF $\beta$-induced proliferation and collagen synthesis in cardiac fibroblasts. Therefore, miR-141 may serve an inhibitory role in cardiac fibrosis.

Bioinformatics analysis revealed that miR-141 bound to the 3'-UTR of SGLT2. The results of luciferase and western blotting assays suggested that miR-141 directly regulated the 
expression of SGLT2. Additionally, SGLT2 overexpression reversed miR-141-mediated reductions of TGF $\beta$-induced proliferation and collagen I and collagen III expression levels in cardiac fibroblasts. Therefore, the present study suggested that there was an association between miR-141 and SGLT2 in the pathogenesis of cardiac fibrosis.

In summary, the present study indicated that SGLT2 expression was upregulated in cardiac fibrosis, and that SGLT2 knockdown reduced cardiac fibrosis and improved cardiac function following MI. Additionally, the results suggested that SGLT2 was regulated by miR-141 in the pathogenesis of cardiac fibrosis. Therefore, the results of the present study provided evidence that the miR-141/SGLT2 axis may serve as a novel target for the treatment of cardiac fibrosis.

\section{Acknowledgements}

Not applicable.

\section{Funding}

No funding was received.

\section{Availability of data and materials}

The datasets used and/or analyzed during the current study are available from the corresponding author on reasonable request.

\section{Authors' contributions}

$\mathrm{CZ}$ and GL conceived, designed and performed the experiments. SF analyzed the data. GL wrote the manuscript. All authors read and approved the final manuscript.

\section{Ethics approval and consent to participate}

All experimental procedures were approved by the Animal Ethics Committee of the Second Affiliated Hospital of Wannan Medical College, Wuhu, China (approval no. DWL-1804-007).

\section{Patient consent for publication}

Not applicable.

\section{Competing interests}

The authors declare that they have no competing interests.

\section{References}

1. Mcmanus DD, Gore J, Yarzebski J, Spencer F, Lessard D and Goldberg RJ: Recent trends in the incidence, treatment, and outcomes of patients with STEMI and NSTEMI. Am J Med 124: 40-47, 2011.

2. Pontecorboli G, Ventura RMFI, Carlosena A, Benito EM, Pratgonzales S, Padeletti L and Mont L: Use of delayed-enhancement magnetic resonance imaging for fibrosis detection in the atria: A review. Europace 19: 180-189, 2017.

3. Nagalingam RS, Safi HA and Czubryt MP: Gaining myocytes or losing fibroblasts: Challenges in cardiac fibroblast reprogramming for infarct repair. J Mol Cell Cardiol 93: 108-114, 2016.
4. Tahrani AA, Barnett AH and Bailey CJ: SGLT inhibitors in management of diabetes. Lancet Diabetes Endocrinol 1: 140-151, 2013.

5. Wells RG, Mohandas TK and Hediger MA: Localization of the $\mathrm{Na}+$ /glucose cotransporter gene SGLT2 to human chromosome 16 close to the centromere. Genomics 17: 787-789, 1993.

6. Kashiwagi Y, Nagoshi T, Yoshino T, Tanaka TD, Ito K, Harada T, Takahashi H, Ikegami M, Anzawa R and Yoshimura M: Expression of SGLT1 in human hearts and impairment of cardiac glucose uptake by phlorizin during ischemia-reperfusion injury in mice. PLoS One 10: e0130605, 2015.

7. Koepsell H: The Na(+)-D-glucose cotransporters SGLT1 and SGLT2 are targets for the treatment of diabetes and cancer. Pharmacol Ther 170: 148-165, 2017.

8. Furtado RHM, Bonaca MP, Raz I, Zelniker TA, Mosenzon O, Cahn A, Kuder J, Murphy SA, Bhatt DL, Leiter LA, et al: Dapagliflozin and cardiovascular outcomes in patients with type 2 diabetes mellitus and previous myocardial infarction. Circulation 139: 2516-2527, 2019.

9. Reid J, Rana K, Niman S, Sheikh-Ali M, Lewis T, Choksi RR and Goldfaden RF: Sodium-glucose cotransporter-2 (SGLT-2) inhibitors for cardiovascular disease prevention. Am J Cardiovasc Drugs 20: 419-429, 2020.

10. Zinman B, Wanner C, Lachin JM, Fitchett D, Bluhmki E, Hantel S, Mattheus M, Devins T, Johansen OE, Woerle HJ, et al: Empagliflozin, cardiovascular outcomes, and mortality in type 2 diabetes. N Engl J Med 373: 2117-2128, 2015.

11. Habibi J, Aroor AR, Sowers JR, Jia G, Hayden MR, Garro M, Barron BJ, Mayoux E, Rector RS, Whaley-Connell A and DeMarco VG: Sodium glucose transporter 2 (SGLT2) inhibition with empagliflozin improves cardiac diastolic function in a female rodent model of diabetes. Cardiovasc Diabetol 16: 9, 2017.

12. Ye Y, Bajaj M, Yang HC, Perez-Polo JR and Birnbaum Y: SGLT-2 inhibition with dapagliflozin reduces the activation of the Nlrp3/ASC inflammasome and attenuates the development of diabetic cardiomyopathy in mice with type 2 diabetes. Further augmentation of the effects with saxagliptin, a DPP4 inhibitor. Cardiovasc Drugs Ther 31: 119-132, 2017.

13. Piccoli MT, Bar C and Thum T: Non-coding RNAs as modulators of the cardiac fibroblast phenotype. J Mol Cell Cardiol 92: 75-81, 2016.

14. Wang H, Zhang X, Li Y, Ma Y, Zhang Y, Liu Z, Zhou J, Lin Q, Wang Y, Duan $\mathrm{C}$ and Wang C: Improved myocardial performance in infarcted rat heart by co-injection of basic fibroblast growth factor with temperature-responsive chitosan hydrogel. J Heart Lung Transplant 29: 881-887, 2010.

15. Yuan J, Chen H, Ge D, Xu Y, Xu H, Yang Y, Gu M, Zhou Y, Zhu J, Ge T, et al: Mir-21 promotes cardiac fibrosis after myocardial infarction via targeting Smad7. Cell Physiol Biochem 42: 2207-2219, 2017

16. Hao K, Lei W, Wu H, Wu J, Yang Z, Yan S, Lu XA, Li J, Xia X, Han X, et al: LncRNA-Safe contributes to cardiac fibrosis through Safe-Sfrp2-HuR complex in mouse myocardial infarction. Theranostics 9: 7282-7297, 2019.

17. Livak KJ and Schmittgen TD: Analysis of relative gene expression data using real-time quantitative PCR and the 2(-Delta Delta C(T)) method. Methods 25: 402-408, 2001.

18. Wang H, Qi C and Wan D: MicroRNA-377-3p targeting MMP-16 inhibits ovarian cancer cell growth, invasion, and interstitial transition. Ann Transl Med 9: 124, 2021.

19. Guo J, Gan Q, Gan C, Zhang X, Ma X and Dong M: LncRNA MIR205HG regulates melanomagenesis via the miR-299-3p/VEGFA axis. Aging (Albany NY) 13: 5297-5311, 2021.

20. Wu H, Zhou X, Wang X, Cheng W, Hu X, Wang Y, Luo B, Huang $\mathrm{W}$ and $\mathrm{Gu} \mathrm{J}$ : miR-34a in extracellular vesicles from bone marrow mesenchymal stem cells reduces rheumatoid arthritis inflammation via the cyclin I/ATM/ATR/p53 axis. J Cell Mol Med 25: 1896-1910, 2021

21. Qu X, Du Y, Shu Y, Gao M, Sun F, Luo S, Yang T, Zhan L, Yuan Y, Chu W, et al: MIAT is a pro-fibrotic long non-coding RNA governing cardiac fibrosis in post-infarct myocardium. Sci Rep 7: 42657, 2017.

22. Zhou B and Yu JW: A novel identified circular RNA, circRNA_010567, promotes myocardial fibrosis via suppressing miR-141 by targeting TGF- $\beta 1$. Biochem Biophys Res Commun 487: 769-775, 2017.

23. Travers JG, Kamal FA, Robbins J, Yutzey KE and Blaxall BC: Cardiac fibrosis: The fibroblast awakens. Circ Res 118: 1021-1040, 2016. 
24. Moore-Morris T, Guimaraes-Camboa N, Yutzey KE, Puceat M and Evans SM: Cardiac fibroblasts: From development to heart failure. J Mol Med (Berl) 93: 823-830, 2015.

25. Leask A: Getting to the heart of the matter new insights into cardiac fibrosis. Circ Res 116: 1269-1276, 2015.

26. Schelbert EB, Fonarow GC, Bonow RO, Butler J and Gheorghiade M: Therapeutic targets in heart failure: Refocusing on the myocardial interstitium. J Am Coll Cardiol 63: 2188-2198, 2014.

27. Okada J, Yamada E, Saito T, Yokoo H, Osaki A, Shimoda Y, Ozawa A, Nakajima Y, Pessin JE, Okada S and Yamada M: Dapagliflozin inhibits cell adhesion to collagen I and IV and increases ectodomain proteolytic cleavage of DDR 1 by increasing ADAM10 activity. Molecules 25: 495, 2020.

28. Grimaldi V, De Pascale MR, Zullo A, Soricelli A, Infante T, Mancini FP and Napoli C: Evidence of epigenetic tags in cardiac fibrosis. J Cardiol 69: 401-408, 2017.
29. Cheng R, Dang R, Zhou Y, Ding M and Hua H: MicroRNA-98 inhibits TGF- $\beta 1$-induced differentiation and collagen production of cardiac fibroblasts by targeting TGFBR1. Human Cell 30: 192-200, 2017.

30. Van Rooij E, Sutherland LB, Thatcher JE, Dimaio JM, Naseem RH, Marshall WS, Hill JA and Olson EN: Dysregulation of microRNAs after myocardial infarction reveals a role of miR-29 in cardiac fibrosis. Proc Natl Acad Sci USA 105: 13027-13032, 2008.

31. Li L, Bounds KR, Chatterjee P and Gupta S: MicroRNA-130a, a potential antifibrotic target in cardiac fibrosis. J Am Heart Assoc 6: e006763, 2017.

(i) (1) () This work is licensed under a Creative Commons cc) Attribution-NonCommercial-NoDerivatives 4.0 International (CC BY-NC-ND 4.0) License. 